\title{
DESIGN OF A COMPACT HIGH CURRENT DENSITY INJECTOR FOR HEAVY ION FUSION*
}

\author{
J. W. Kwan, E. Henestroza, Lawrence Berkeley National Laboratory, \\ L. Ahle, D.P. Grote, Lawrence Livermore National Laboratory
}

\begin{abstract}
A compact multiple-beam injector concept is being developed for heavy ion inertial fusion. The design is based on merging high intensity miniature beamlets of a few $\mathrm{mA}$ each to form a large beam. In order to minimize emittance growth, the beamlets are kept separated in grids until they are allowed to merge together at $1.2 \mathrm{MeV}$. With optimized positioning and aiming, the merged beam can quickly match into an electrostatic quadruple (ESQ) channel. Simulation results have shown that, based on this design, a $1.6 \mathrm{MeV}, 0.5 \mathrm{~A}$ beam of $\mathrm{K}+$ ions at the end of the injector has a normalized emittance of approximately $1.0 \pi$-mm-mrad which is comparable to that from the previous low current density design. The advantage is a factor of 6 reduction in the combined injector and matching section length
\end{abstract}

\section{INTRODUCTION}

For heavy ion driven inertial fusion (HIF) induction linac drivers, a typical injector requires total beam current of 50-100 A and is comprised of many individual beams of $\approx 0.5 \mathrm{~A}$ each. Heavy ion beams have significant spacecharge effects, so the current per beam is limited by the focusing capabilities of the beam extraction and transport systems. In our previous paper [1], we have presented an injector design of an array of beams feeding into the channels of a multiple-beam induction linac. However we would like to improve on that design because of its enormous size, and not so perfect beam quality. In this paper, we present an alternate concept that results in a smaller size injector and good beam quality.

\section{TWO DIFFERENT APPROACHES}

In our injector design studies, we have found that the LEBT physics dominates the injector requirements and consequently dictates the types of ion sources that can be used in the system. Based on high voltage breakdown scaling (which scales poorly for large beams), the current density is low when the beam current is large at low energy. Thus one way to obtain large current is to use a large aperture ion source. This is the traditional approach for HIF injector; the ELISE (single-beam ILSE) injector was built that way. The ions are extracted by a diode followed by an ESQ accelerator and a long matching

* This work is supported by the Office of Fusion Energy Science, US DOE under contract No. DE-AC03-76SF00098 (LBNL) and W-7405-ENG-48 (LLNL).

Email: jwkwan@1bl.gov section (the purpose of a matching section is to compress the beam size and transform a round beam from an ion gun into an elliptical beam for an ESQ lattice). Since $J$ is low in this case, the effective ion temperature must be very low in order to achieve high brightness. The only known type of ion source that can simultaneously meet the requirements of low ion temperature and a well-defined large emitting surface is the alkaline metal surface ionization source.

A preliminary conceptual design of a driver scale multiple-beam injector based on this concept is shown in Fig. 1.[1] The figure shows the outer-most beamline in a typical driver scale multiple beam injector. It is not considered as an attractive design because such a large injector is difficult to build, has large stored electrical energies and will be prohibitively expensive (not on an affordable development path). Furthermore, bending the beams in the ESQ channel using specially shaped electrodes can introduce significant aberrations. Nevertheless, this approach may still be a viable solution for near-term experiments requiring a single-beam injector (without bending).

The second approach for designing HIF injector is to achieve brightness by maximizing the current density. Such a system starts with an array of high current density mini-beamlets (of a few $\mathrm{mA}$ each) that are kept separated from each other within a set of acceleration grids in order to minimize the space charge expansion. After they have gained sufficient kinetic energy $(>1.2 \mathrm{MeV}$ ), the minibeamlets will be allowed to merge together to form a high current beam. The grids are designed to aim the minibeamlets differently in the two transverse planes in such a way that beam matching into an ESQ channel can be achieved automatically during the merging stage. Ultimately, the brightness depends on the average current density (including the grid transparency) and the emittance of the merged beam. With a high average current density, the source end of the injector can be similar in size comparing to the ESQ array at the beginning of the linac such that the multiple beamlines are nearly parallel and no (or minimum) bending is required. Figure 2 is a schematic diagram of an injector beamline based on this concept.

As shown in the figure, the total length from the ion source to the end of merging/matching section is only about $1 \mathrm{~m}$. Comparing figures 1 and 2, we found a factor of 6 reduction in size using the new approach. Likewise, we expect similar reduction in cost as well. Thus 


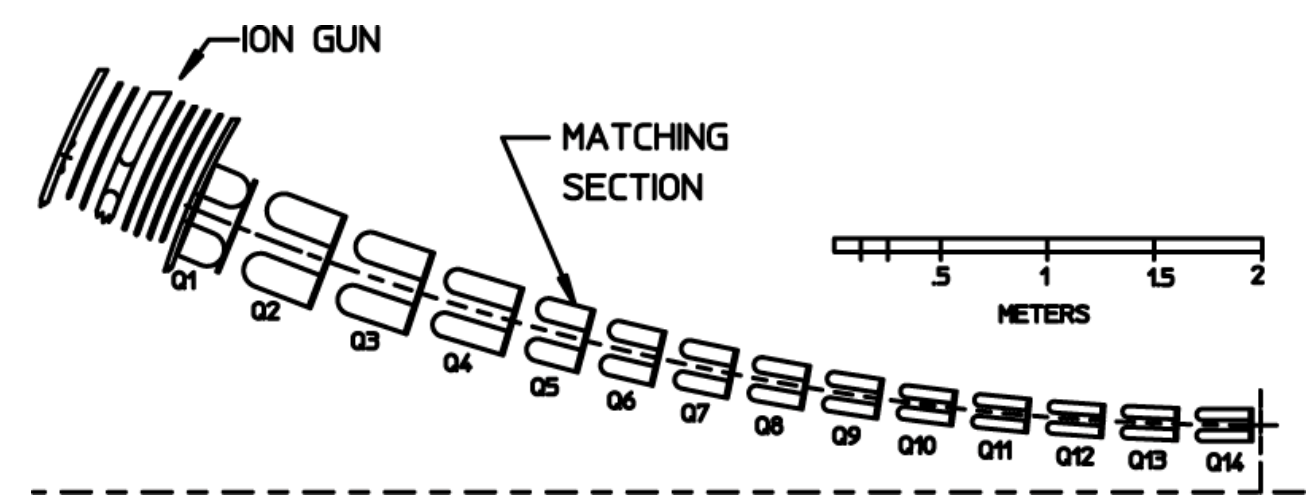

Figure 1: Schematic diagram of a single beamline at the edge of the injector array based on large aperture source.

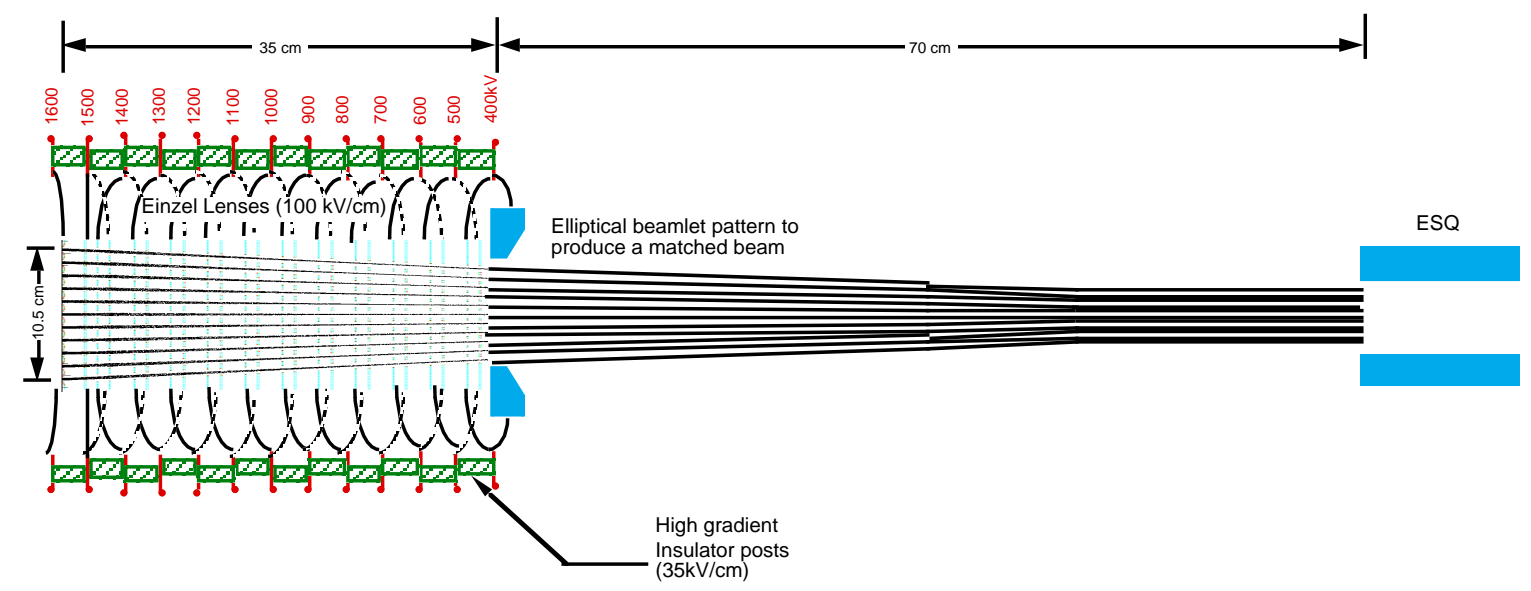

Fig. 2. Schematic diagram of an injector beamline based on merging mini-beamlets.

the high $\mathrm{J}$ mini-beamlets approach provides a possible affordable development path for a driver scale multiplebeam injector.

\section{A COMPACT INJECTOR DESIGN}

Since the current density of the mini-beamlets is on the order of $100 \mathrm{~mA} / \mathrm{cm}^{2}$, it is necessary to use accel-decel Einsel lens focussing during the first $1 \mathrm{MV}$ of acceleration. Figure 2 illustrates the schematic of how to provide voltages to the grids. The acceleration voltage is evenly divided into $100 \mathrm{kV}$ per step and each voltage step will be applied to a pair of acceleration grids. Each upstream grid has openings for spokes to penetrate and support a downstream grid at a higher voltage. The local voltage gradient is about $100 \mathrm{kV} / \mathrm{cm}$ at the gap between grids and $34 \mathrm{kV} / \mathrm{cm}$ at the region where high gradient insulator posts are identified in the figure. Recent development at LLNL has suggested that multi-layered insulator can be made to withstand such gradient. Testing is being arranged to confirm its performance in the near future. The small size insulator posts are used only to reinforce support for the acceleration grid structure and is not for the vacuum enclosure. The entire acceleration structure containing multiple beamlines must be mounted inside a vacuum vessel. Depending on the method to be determined later, the vacuum vessel can be a metal tank with high voltage feed-through or an insulator column.

The main physics issues involved in the mini-beamlets approach are emittance growth and envelope matching in the merging process. Our design incorporates 3 features to minimize the emittance growth: (1) use a large number of beamlets ( $\geq 100)$; (2) let the beamlet merging takes place at energy $\geq 1.2 \mathrm{MeV}$; (3) geometrically aim the beamlets inward to produce a strong focussing effect and to minimize the length of the merging region. Figure 3 shows the beam envelopes produced by an elliptical array of mini-beamlets matching into an ESQ channel. The emittance diagram from merging 100 beamlets is shown in Figure 4. The rms emittance growth and how it reaches equilibrium in about $10 \mathrm{~m}$ after entering the ESQ channel in the linac is shown in Fig. 5.

In the simulation, the 100 beamlets have a total current of $0.5 \mathrm{~A}$ and a transparency of $7 \%$ of the grid area. Table 1 shows the emittance growth calculated for various beamlet initial emittance. The final beam emittance is $0.85 \pi$-mmmrad if each beamlet has an initial emittance of $0.005 \pi$ $\mathrm{mm}$-mrad. Interestingly when the initial beamlet 


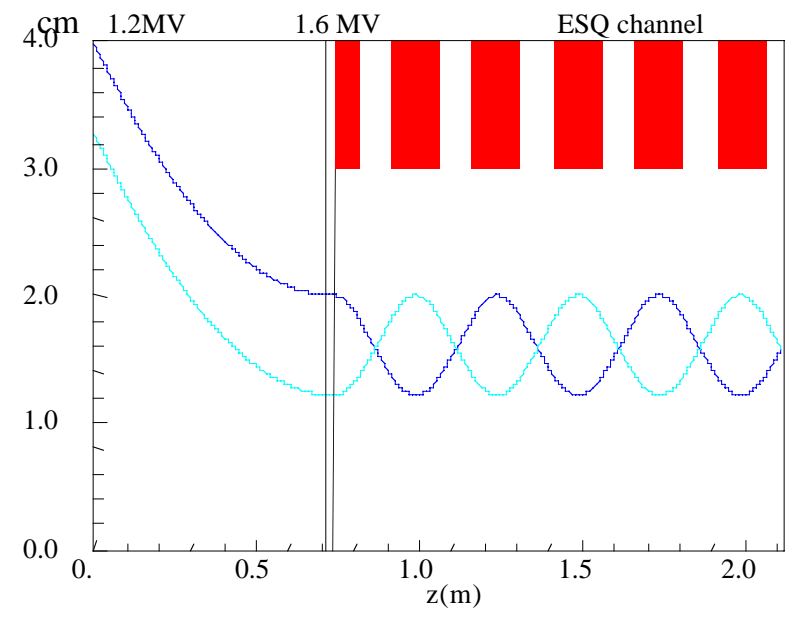

Figure 3: Beam envelopes of merged 100 beamlets.

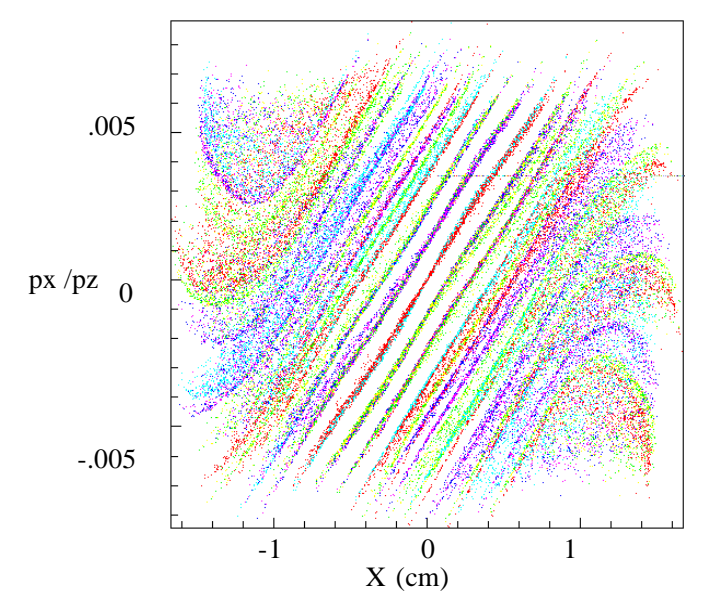

Fig. 4. Emittance diagram of the merged beamlets.

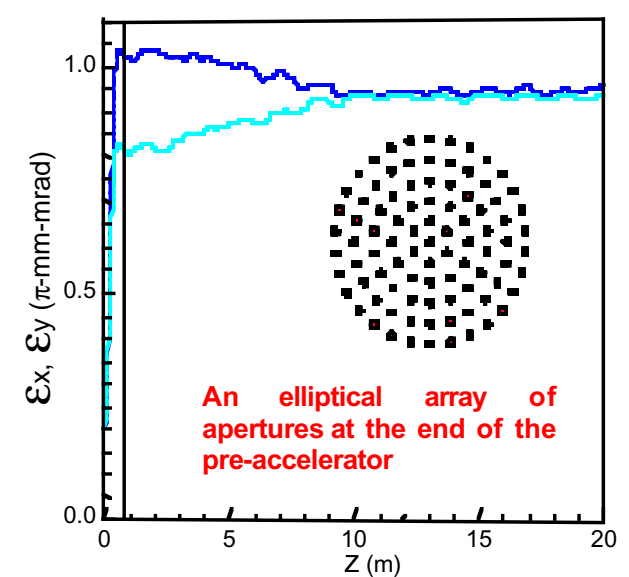

Fig. 5. Emittance growth due to merging beamlets

Table 1.Emittance of merging 100 beamlets, $5 \mathrm{~mA}$ each.

\begin{tabular}{|c|c|}
\hline $\begin{array}{c}\text { Initial emittance } \\
\text { (pi-mm-mrad) }\end{array}$ & $\begin{array}{c}\text { Final emittance } \\
\text { (pi-mm-mrad) }\end{array}$ \\
\hline 0.005 & 0.85 \\
\hline 0.01 & 1.0 \\
\hline 0.02 & 1.2 \\
\hline
\end{tabular}

emittance is raised to $0.01 \pi$-mm-mrad and $0.02 \pi$-mm$\mathrm{mrad}$, the final emittance only increases to $1.0 \pi$-mmmrad and $1.2 \pi$-mm-mrad respectively. This effect simply means the emittance growth is dominated by the space charge effect in the merging process and is not very sensitive to the initial ion temperature. We have not determined whether the $7 \%$ beam occupancy or the number of 100 beamlets are the optimum value to achieve the highest possible brightness.

\section{ION SOURCE OPTIONS}

Since the final emittance increases only weakly with the initial emittance of the beamlets (see table 1), a significant advantage for this approach is the possibility of employing ion sources that produce ions with higher temperature. For example, we have already demonstrated that an rf-driven gas plasma source can easily produce a current density $>100 \mathrm{~mA} / \mathrm{cm}^{2}$ of argon ions.[2] For gas plasma sources we must consider the effect of charge exchange at the region below a few hundred $\mathrm{keV}$ where the cross-section is large. Charge exchange produces slower moving ions and therefore energy dispersion on the beam energy. Thus, it is necessary to operate the gas plasma source with minimum gas pressure as well as design the accelerator grids for maximum conductance.

\section{OTHER ENGINEERING ISSUES}

Clearly for high net beam brightness, it is important to develop high transparency accelerator grids and also be able to operate the grids at high enough voltage gradient to focus the beamlets. These engineering issues are similar to that of neutral beam injectors for magnetic fusion devices. In fact, an experiment merging 19 beamlets into an ESQ channel has been done before in neutral beam development [3].

\section{SUMMARY}

We have presented an alternate conceptual design of multiple beam injector for HIF. It makes use of minibeamlets to achieve high current density, and merging the beamlets to produce large total current. Simulation has shown that the emittance growth from merging is acceptable. The most important feature from the new approach is the drastic reduction in the size of a multiple beam driver scale injector. Experiments are planned to begin this summer to confirm our design and validate this concept.

\section{REFERENCES}

[1] J.W. Kwan, et al, Proc. Particle Accelerator Conf., New York, March, (1999), p.1937

[2] L. Ahle, et al, "RF gas plasma source development for heavy ion fusion", this proceedings.

[3] J.W. Kwan, et al, Rev. Sci. Instrum, 66(7), p3864, (1995). 